\title{
Clear cell renal cell carcinoma associated microRNA expression signatures identified by an integrated bioinformatics analysis
}

\author{
Jiajia Chen ${ }^{1,2 \dagger}$, Daqing Zhang ${ }^{1+}$, Wenyu Zhang ${ }^{1}$, Yifei Tang ${ }^{1}$, Wenying Yan ${ }^{1}$, Lingchuan Guo ${ }^{3}$ and Bairong Shen ${ }^{1 *}$
}

\begin{abstract}
Background: Clear cell renal cell carcinoma (ccRCC) represents the most invasive and common adult kidney neoplasm. Mounting evidence suggests that microRNAs (miRNAs) are important regulators of gene expression. But their function in tumourigenesis in this tumour type remains elusive. With the development of high throughput technologies such as microarrays and NGS, aberrant miRNA expression has been widely observed in ccRCC. Systematic and integrative analysis of multiple microRNA expression datasets may reveal potential mechanisms by which microRNAs contribute to $\mathrm{CCRCC}$ pathogenesis.

Methods: We collected 5 public microRNA expression datasets in cCRCC versus non-matching normal renal tissues from GEO database and published literatures. We analyzed these data sets with an integrated bioinformatics framework to identify expression signatures. The framework incorporates a novel statistic method for abnormal gene expression detection and an in-house developed predictor to assess the regulatory activity of microRNAs. We then mapped target genes of DE-miRNAs to different databases, such as GO, KEGG, GeneGo etc, for functional enrichment analysis.
\end{abstract}

Results: Using this framework we identified a consistent panel of eleven deregulated miRNAs shared by five independent datasets that can distinguish normal kidney tissues from ccRCC. After comparison with 3 RNA-seq based microRNA profiling studies, we found that our data correlated well with the results of next generation sequencing. We also discovered 14 novel molecular pathways that are likely to play a role in the tumourigenesis of ccRCC.

Conclusions: The integrative framework described in this paper greatly improves the inter-dataset consistency of microRNA expression signatures. Consensus expression profile should be identified at pathway or network level to address the heterogeneity of cancer. The DE-miRNA signature and novel pathways identified herein could provide potential biomarkers for $\mathrm{CCRCC}$ that await further validation.

Keywords: Meta-analysis, Network biomarker, MicroRNA, Clear cell renal cell carcinoma, Pathway analysis, Heterogeneity

\section{Background}

Renal cell carcinoma (RCC) represents the leading cause of death among urological malignancies [1]. Clear cell renal cell carcinoma (ccRCC) is the most common histological subtype of RCC. Early stage of renal cancers do not usually cause symptom and it's difficult to establish an accurate diagnose. ccRCC is relatively resistant to chemotherapy or

\footnotetext{
*Correspondence: bairong.shen@suda.edu.cn

${ }^{\dagger}$ Equal contributors

'Center for Systems Biology, Soochow University, Suzhou 215006, China Full list of author information is available at the end of the article
}

radiotherapy [2] and the overall clinical outcome is poor [3]. Thus the need for diagnostic and prognostic biomarkers in ccRCC is urgent. Nevertheless, to our knowledge there are still no biomarkers in routine clinical practice in ccRCC.

microRNAs are single-stranded, non-coding RNAs that regulate gene expression at the post-transcriptional level [4]. Aberrant changes in microRNA expression have been shown to be associated with human malignancies [5,6]. Various studies have investigated the miRNA profile in ccRCC lesions in comparison to non-tumoral kidney tissues by microarray technologies [7-13], RT-qPCR [14-16] and

\section{Biomed Central}

(c) 2013 Chen et al.; licensee BioMed Central Ltd. This is an Open Access article distributed under the terms of the Creative Commons Attribution License (http://creativecommons.org/licenses/by/2.0), which permits unrestricted use, distribution, and reproduction in any medium, provided the original work is properly cited. 
more recently with next generation sequencing [17-19]. Altered expression of miRNAs in ccRCC has been frequently reported. Nevertheless, the DE-miRNA lists from different laboratories vary widely due to the inter-platform differences and the limited sample sizes. Potential mechanisms by which miRNAs contribute to ccRCC pathogenesis are still poorly understood.

Cancer is a systems biology disease, therefore the biomarkers discovery should take into account the heterogeneity and complexity of carcinogenesis. There is a growing movement from individual marker discovery to a systemsoriented paradigm. Due to this recognition, we describe herein an integrated bioinformatics approach to obtain a consistent microRNA expression signature as well as novel microRNA-regulated molecular pathways that contribute to the pathogenesis of ccRCC. The analysis pipeline of this paper is outlined in Figure 1.

\section{Methods}

\section{Dataset collection}

Five publicly available microRNA expression datasets on ccRCC versus non-matching normal renal tissues were downloaded, four of them from the GEO (Gene Expression Omnibus) database, and one from supplementary materials of published literature. Table 1 gives detailed information of the miRNA expression datasets including the original statistical methods used for the DE-miRNA identification. Four mRNA expression datasets by Affymetrix arrays were also extracted from GEO (detailed information given as Additional file 1). All the datasets are downloaded in raw data file format. Probe sequences were mapped to Sanger miRBase 18 [20] for unified miRNA names.

\section{Data processing}

The quantified probing signals were background corrected using Normexp, with offset value set as 0 . The backgroundsubtracted data were normalized using the Quantile algorithm. Averages were derived from all quantile normalized data per miRNA for statistical analysis. Missing data were imputed with the k-nearest neighbors imputation approach $(\mathrm{k}=5)$. We wrote the $\mathrm{R}$ scripts to run the processing procedures for all datasets. The mRNA expression data were analyzed using affy package from Bioconductor with RMA method. For the gene annotation, genes that correspond to

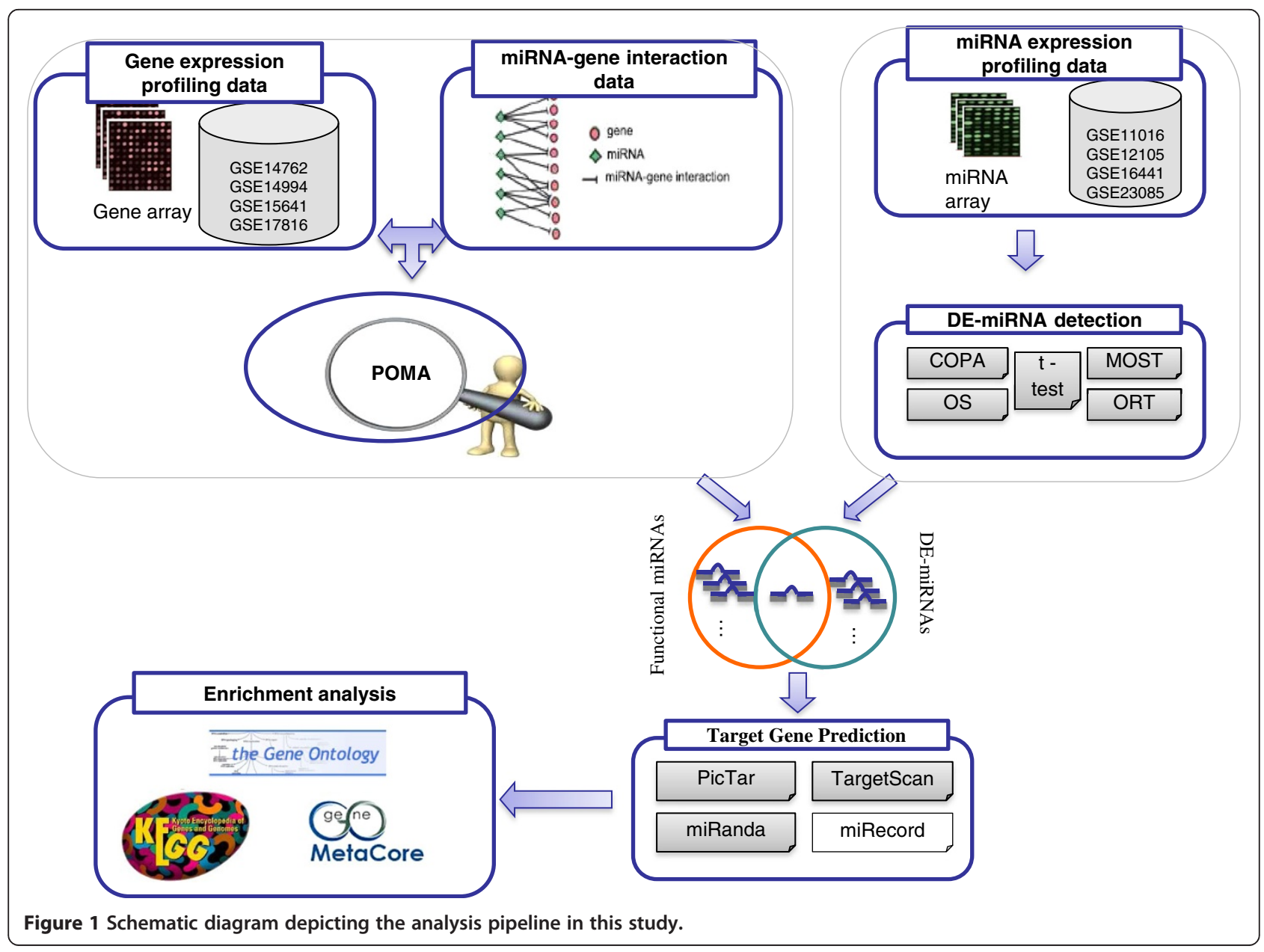


Table 1 Summary of microRNA expression datasets used in this study

\begin{tabular}{|c|c|c|c|c|c|c|}
\hline \multirow{2}{*}{$\begin{array}{c}\text { GEO } \\
\text { accession }\end{array}$} & \multirow[t]{2}{*}{ Reference } & \multirow[t]{2}{*}{ Platforms } & \multirow{2}{*}{$\begin{array}{l}\text { Probe } \\
\text { Number }\end{array}$} & \multicolumn{2}{|c|}{ Number of samples } & \multirow[t]{2}{*}{ Statistics } \\
\hline & & & & normal tissue & cancer tissue & \\
\hline GSE11016 & [6] & LC MRA-1001 & 835 & 17 & 17 & t-test \\
\hline GSE12105 & [7] & Agilent Human microRNA Microarray & 490 & 12 & 12 & t-test \\
\hline GSE16441 & [8] & Agilent Human microRNA Microarray & 851 & 8 & 8 & SAM \\
\hline GSE23085 & [10] & LC MRA-1001 & 881 & 20 & 20 & t-test \\
\hline Weng & [18] & Agilent Human microRNA Microarray & 723 & 3 & 3 & t-test \\
\hline
\end{tabular}

multiple probes were removed and those with unique probe were retained for further analysis.

\section{Comparison of the outlier detection methods}

We compared the performance of five prevailing outlier detection algorithms, COPA (Cancer Outlier Profile Analysis) [21], MOST (Maximum Ordered Subset T-statistics) [22], ORT (Outlier Robust T-statistics) [23], OS (Outlier Sum) [24] and t-test.

All the algorithms were implemented in R scripts written by Lian [22] and Wang [25]. Outliers for each expression dataset were determined by five methods respectively. The threshold for outlier detection was set 0.05 (5\%) for all the methods. The outliers detected by at least 3 methods were taken as the putative DE-miRNAs. The proportion of the putative DE-miRNAs in the outlier list found by each method was then calculated. Median value of the percentage among 5 datasets was then calculated as the accuracy for each method.

\section{Determination of the differentially expressed microRNAs and mRNAs}

Outlier microRNAs and mRNAs were detected with MOST, implemented in R scripts by Lian et al. [22]. Outliers that rank top $5 \%$ were extracted as differential candidates.

\section{Target gene prediction for DE-microRNAs}

The mRNAs targeted by the DE-microRNAs were obtained from three target prediction algorithms (TargetScan [26], PicTar [27] and miRanda [28]) as well as a database with experimental evidence (miRecord [29]). In order to obtain a more reliable result, we removed the targets found by only one prediction programs.

\section{Functional enrichment of DE-miRNA targets}

We then mapped the target genes of DE-miRNAs to different databases, such as GO (Gene Ontology), KEGG, GeneGo, for functional enrichment analysis. GO and KEGG pathway enrichment were performed using DAVID Bioinformatics Resources 6.7 [30]. GeneGo pathway analysis was performed by MetaCoreTM (GeneGo Inc). In MetaCoreTM, P-values were calculated by hypergeometric distribution to evaluate the statistical significance of the enriched pathways. MetaCoreTM used FDR (False Discovery Rate) adjustment for multiple test correction.

\section{Results}

Detection of DE-miRNAs with a novel statistical method

T-statistics is most widely used in differential gene expression detection for microarray studies. However, there is a growing realization that the activation patterns of oncogenes are highly heterogeneous. Some oncogenes show altered expressions only in a minor fraction of samples. Tomlins et al. [31] showed that t-statistics has poor detection power for such unconventional circumstance. The problem with t-statistics has motivated a variety of novel analytical methods such as COPA (Cancer Outlier Profile Analysis) [21], MOST (Maximum Ordered Subset T-statistics) [22], ORT (Outlier Robust T-statistics) [23] and OS (Outlier Sum) [24]. In our previous study [32] we have applied these novel methods to prostate cancer microarray datasets. We have demonstrated that the choice of outlier detection method can greatly affect the genes that are identified.

We compared the performance of these methods in outlier detection based on microRNA expression data in ccRCC. Each method was applied to 5 public microRNA profiling datasets (details of these datasets are given in method section) to obtain the outliers. Outliers detected by at least 3 methods were designated as putative DEmiRNAs. The proportion of the putative outliers in the original gene list was then calculated and the median observation among 5 datasets was defined as the accuracy for the method, as illustrated in Figure 2.

Among the five algorithms for comparison, MOST gives best accuracy. ORT also displays favorable performance. COPA performs a little worse than ORT, but still offers some improvement over the t-statistics. The performance of the OS statistics is noticeably worst. The advantage over $\mathrm{t}$-test is apparent for the majority of the novel methods. Thus we come to the conclusion that the newly developed statistics show superior performance in detecting outliers, and therefore provide promising alternatives to the traditional t-statistics. In this study we chose MOST to identify differentially expressed outliers from public datasets. MOST seems to have superior performance when the number of activated samples is unknown [22]. The threshold of 


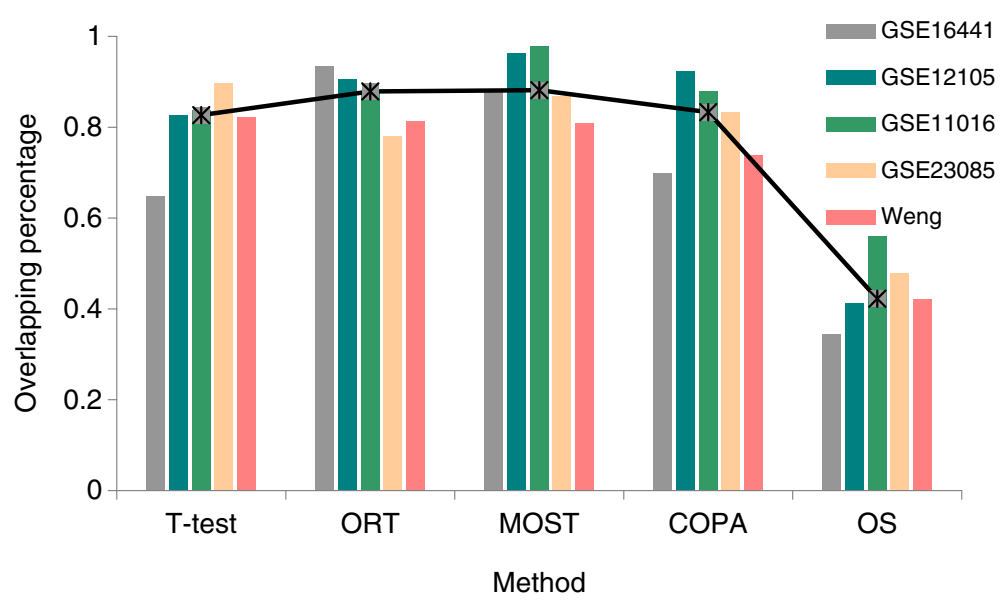

Figure 2 The percentage of the putative outliers in the original gene list by different methods. The overlapping percentage was calculated for 5 datasets respectively. The median value among the 5 datasets was defined as the accuracy for the method.

outliers is set as 0.05 to select the top 5\% of the miRNA outliers.

\section{Refinement of DE-miRNA lists with the Pipeline of Outlier microRNA Analysis (POMA)}

We used an in-house prediction model POMA (Pipeline of Outlier microRNA Analysis) to remove false positive discoveries from the outlier microRNAs detected by MOST. POMA is a model created by our colleagues [33] to evaluate the relevance of microRNAs to given disease conditions. MiRNAs with poor regulatory activity will be excluded from further analysis. POMA is based on two hypotheses: the microRNA activity could be reflected by the deregulated expression of its target genes; if the deregulated genes are targeted exclusively by certain microRNA, that microRNA is more likely to show regulatory activity. The stepwise procedure of POMA is described as follows:

a) We conducted a comprehensive search of all possible microRNA-mRNA interactions for human. Experimentally validated interactions were extracted from 4 databases: miRecords, miRTarbase, miR2Disease and TarBase. Computationally predicted interactions were retrieved from HOCTAR, starBase, and ExprTargetDB. Based on these data, we reconstructed a human microRNAmRNA interaction network.

b) We reanalyzed 4 public gene expression data in ccRCC vs. normal kidney samples, and identified deregulated genes in ccRCC.

c) The deregulated genes detected in step (b) were subsequently mapped to the microRNA-mRNA interaction network established in step (a), to construct a ccRCC-specific microRNA-mRNA interaction sub-network. d) We defined a Z_score to measure the probability of microRNA having regulatory role in ccRCC:

$$
Z_{\text {score }}=\alpha / \beta
$$

$\alpha$ : Number of outlier genes targeted exclusively by a specific microRNA; $\beta$ : Number of all the outlier genes targeted by that microRNA; $(\alpha, \beta>1)$.

Z_score is calculated for each candidate microRNA in the sub-network. Using a threshold of 0.1, we identified a list of miRNAs with potential regulatory role in ccRCC for each mRNA expression dataset. The final list was an overlap of the active microRNA identified in at least 3 of the 4 gene expression datasets.

e) The active microRNA list found in step (d) was crossmatched with the DE-microRNA list of each miRNA dataset. The intersected microRNAs (listed in Additional file 2) were retained for further analysis.

\section{POMA improves inter-dataset consistency}

POMA predicts a list of microRNA that might play regulatory role in ccRCC. These microRNAs serve as a filter that removes the DE-miRNAs without actual function. After filtration, the DE-miRNA list is greatly reduced, resulting in a robust set of functional microRNAs. In addition, a higher overlap between different miRNA datasets was observed. The inter-dataset overlapping percentage before and after POMA filtration is illustrated in Figure 3.

The $\mathrm{p}$-values for the overlapping percentage were 4.38165E-05 by paired t-test, indicating the significance of the difference. Although the number of DE-microRNAs decreased, the consistency between multiple datasets is greatly improved. The enhanced consistency enables us to extract 


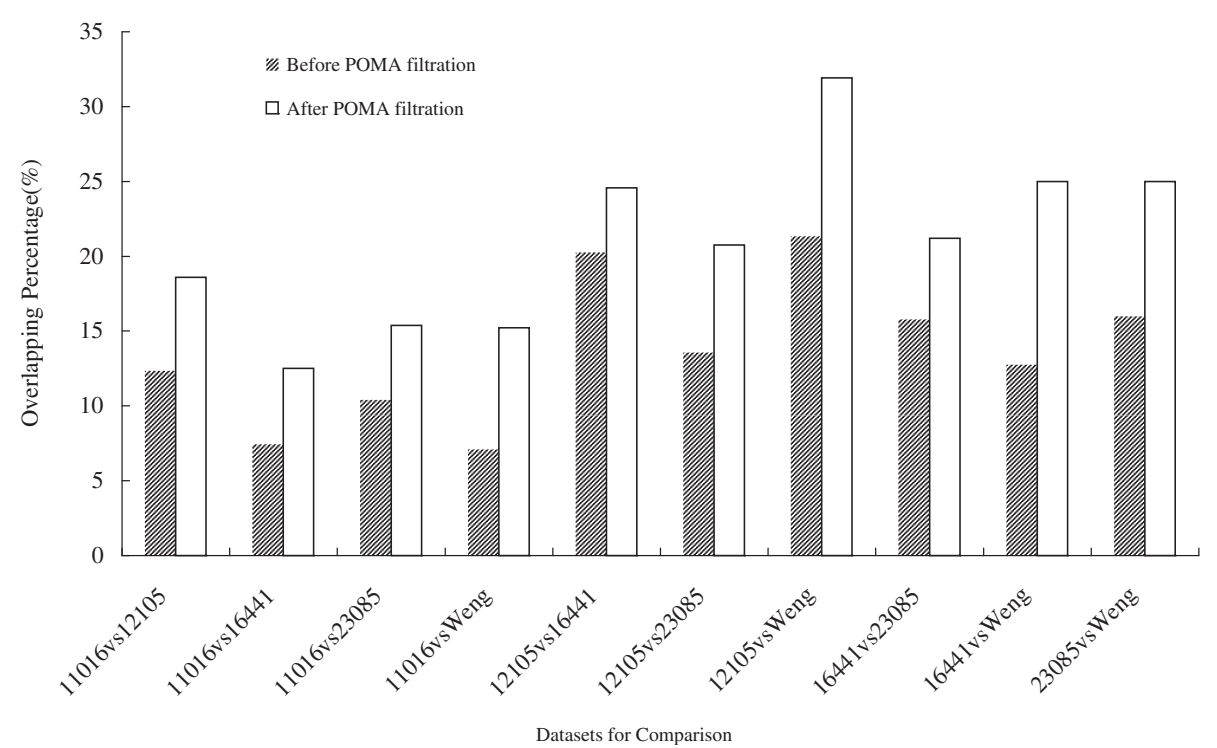

Figure 3 Pair-wise comparison between 5 datasets at different levels. $X$ axis shows the 10 pair-wise comparison sets derived from 5 datasets. $Y$ axis denotes the overlapping percentage at different levels.

common microRNA expression signatures from different datasets. As a result, we retrieved a set of 11 microRNAs (listed in Table 2), which were shared by at least 4 of the 5 datasets.

Furthermore, we performed a literature search of the 11 microRNA markers to validate their relevance in the regulation of ccRCC. It's found that all of them have been reported for their roles in renal cell carcinoma. The numbers of supporting literatures for each microRNA are also given in Table 2. These "literature curated" microRNAs provide a focused and robust signature to separate normal from cancerous kidney tissues.

\section{Targets prediction and functional enrichment}

We conducted a high-stringency target prediction for the DE-miRNAs. Target genes were obtained from both experimentally supported databases and prediction algorithms. Number of target genes for each dataset is listed in Table 3. Detailed list of target genes are available in Additional file 3 . The targets of each individual dataset were mapped to functional databases, e.g. GO [34], KEGG [35] and GeneGo. Table 3 illustrates the number of various biological themes significantly enriched with target genes for each dataset. Detailed lists of the enriched functional categories and pathways could be found in Additional file 4 .

\section{Identification of ccRCC related functions and pathways}

DE-miRNA target genes are statistically enriched in GO terms of cell cycle and transcription. The top enriched GO terms include: regulation of cyclin-dependent protein kinase activity, transcription, DNA-dependent regulation of transcription, regulation of cell proliferation,

Table 2 DE-miRNAs with outlier activity in CCRCC pathogenesis

\begin{tabular}{|c|c|c|c|c|c|c|}
\hline Human microRNA & Accession No. & Chr & Chromosomal location & microRNA cluster & Family & PubMed citation No. \\
\hline hsa-miR-210 & MIMAT0000267 & 11 & transcripts & 0 & 210 & 15 \\
\hline hsa-miR-138-5p & MIMAT0000430 & 3 & intergenic & 0 & 138 & 5 \\
\hline hsa-miR-16-5p & MIMAT0000069 & 13 & transcripts & 1 & 15 & 2 \\
\hline hsa-miR-224-5p & MIMAT0000281 & $x$ & transcripts & 1 & 224 & 9 \\
\hline hsa-miR-34a-5p & MIMAT0000255 & 1 & intergenic & 0 & 34 & 5 \\
\hline hsa-miR-184 & MIMAT0000454 & 15 & intergenic & 0 & 184 & 6 \\
\hline hsa-miR-122-5p & MIMAT0000421 & 18 & intergenic & 1 & 122 & 6 \\
\hline hsa-miR-126-3p & MIMAT0000445 & 9 & transcript & 0 & 126 & 5 \\
\hline hsa-miR-155-5p & MIMAT0000646 & 21 & transcript & 0 & 155 & 14 \\
\hline hsa-miR-15b-5p & MIMAT0000068 & 3 & transcript & 1 & 15 & 3 \\
\hline hsa-miR-660 & MIMAT0003338 & $x$ & transcript & 6 & 188 & 1 \\
\hline
\end{tabular}


Table 3 The number of various enriched biological themes for different datasets

\begin{tabular}{|c|c|c|c|c|c|c|}
\hline Dataset & DE-miRNA & Target & $\begin{array}{c}\text { GO-BP } \\
(\text { FDR }<0.05)\end{array}$ & $\begin{array}{c}\text { GO-MF } \\
(\text { FDR }<0.05)\end{array}$ & $\begin{array}{c}\text { KEGG } \\
\text { FDR }<(0.05)\end{array}$ & $\begin{array}{c}\text { GeneGo } \\
(\text { FDR }<0.001)\end{array}$ \\
\hline GSE 11016 & 21 & 853 & 18 & 9 & 2 & 99 \\
\hline GSE 12105 & 22 & 764 & 54 & 11 & 7 & 152 \\
\hline GSE 16441 & 35 & 1136 & 110 & 15 & 11 & 149 \\
\hline GSE 23085 & 31 & 895 & 53 & 11 & 13 & 125 \\
\hline Weng & 25 & 921 & 67 & 10 & 9 & 135 \\
\hline Shared & 5 & 388 & 8 & 7 & 6 & 62 \\
\hline
\end{tabular}

sequence-specific DNA binding and transcription regulator activity.

KEGG pathways that are significantly enriched with the DE-miRNA targets were also identified, many of which are associated in cancer, e.g. colorectal cancer, Cell cycle, Neurotrophin signaling pathway, Renal cell carcinoma, Prostate cancer, MAPK signaling pathway, and p53 signaling pathway.

The top GeneGo pathway maps regulated by DE-miRNA converge on cell adhesion, cell cycle and cytoskeleton remodeling, most of which are known to play a part in tumor development. Among the significantly enriched GeneGo pathways (FDR < 0.001), 60 were shared by all of the 5 datasets (see Additional file 4). To evaluate the relevance of these pathway maps in ccRCC, we searched PubMed for the published papers describing their constituent network objects in ccRCC. The network objects with previous literature support were considered to be ccRCC-related.

After text mining, 36 out of the 60 pathways (60\%) were found to be highly saturated with well-characterized ccRCCrelated objects (enrichment ratio $>0.15$, p-value $<0.0001$ ). To visualize the most significantly enriched pathways we constructed a scatter plot (Figure 4) by plotting the $-\log 10$ of $\mathrm{p}$-value versus gene enrichment ratio on the $\mathrm{y}$ - and $\mathrm{x}$ axes, respectively. The most meaningful points that display both large enrichment ratio $(>0.15, x$-axis) as well as high statistical significance $(\mathrm{P}<0.0001, \mathrm{y}$-axis $)$ were shown in red. These points could be the potential regulatory pathways in renal carcinogenesis. The top 10 significant GeneGo pathways enriched with ccRCC-related objects are listed in Table 4.

A further PubMed search highlighted 22 out of the 36 putative ccRCC-related pathways with literature support in ccRCC pathogenesis. The remaining 14 pathways without previous annotation are considered to be promising novel pathways contributing to ccRCC (See details in Additional file 5). Among the novel GeneGo pathways, TGF, WNT and cytoskeletal remodeling is most significantly enriched. The pathway is highly saturated with network objects previously found to associate with RCC, such as TCF, AKT, VEGF-A, WNT, Frizzled, TGF-beta, RhoA, Beta-catinin, c-Myc, Cyclin D1 and c-Jun. This pathway focuses on WNT protein family and its downstream effectors. The WNT signaling pathway plays a central role

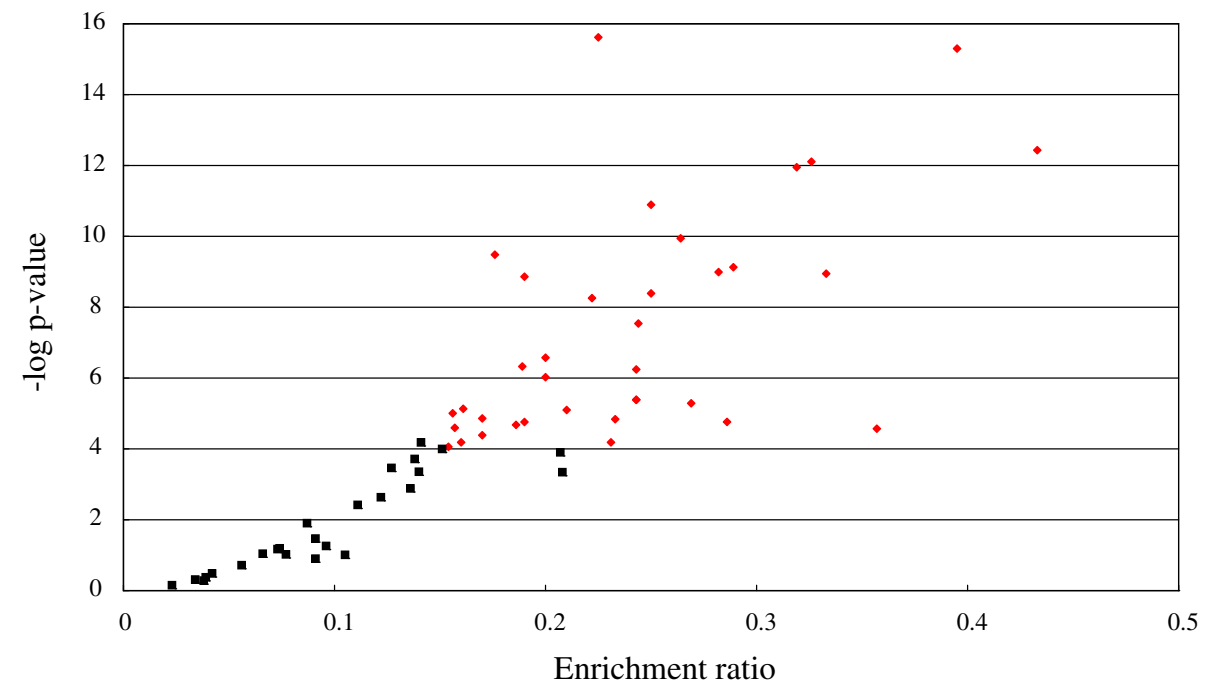

Figure 4 Volcano plot of pathways enriched with RCC-related genes. The red points indicate pathways of interest that display both large enrichment ratio $(>0.15, x$-axis) as well as high statistical significance $(P<0.0001, y$-axis). 
Table 4 Top 10 of the significant GeneGo pathways enriched with both DE-miRNA targets and RCC-related genes

\begin{tabular}{|c|c|c|c|c|}
\hline Pathway map & $\begin{array}{c}\text { Pathway map } \\
\text { category }\end{array}$ & $\begin{array}{c}\text { Ratio of RCC related } \\
\text { objects }\end{array}$ & P-value & PubMed citation count \\
\hline TGF, WNT and cytoskeletal remodeling & Cytoskeleton remodeling & $25 / 111$ & $2.41 \mathrm{E}-16$ & \\
\hline AKT signaling & Signal transduction & $17 / 43$ & $5.00 \mathrm{E}-16$ & 70 \\
\hline Brca1 as a transcription regulator & DNA damage & $13 / 30$ & $3.70 \mathrm{E}-13$ & \\
\hline PTEN pathway & Signal transduction & $15 / 46$ & 7.79E-13 & 22 \\
\hline PIP3 signaling in cardiac myocytes & Development & $15 / 47$ & $1.12 \mathrm{E}-12$ & \\
\hline Regulation of epithelial-to-mesenchymal transition (EMT) & Development & $16 / 64$ & $1.12 \mathrm{E}-11$ & 12 \\
\hline Influence of Ras and Rho proteins on G1/S Transition & Cell cycle & $14 / 53$ & $1.14 \mathrm{E}-10$ & 2 \\
\hline Cytoskeleton remodeling & Cytoskeleton remodeling & $18 / 102$ & 3.30E-10 & 2 \\
\hline Regulation of G1/S transition (part 1) & Cell cycle & $11 / 38$ & 7.43E-10 & 4 \\
\hline Receptor-mediated HIF regulation & Transcription & $11 / 39$ & $1.02 \mathrm{E}-9$ & 7 \\
\hline
\end{tabular}

during tumorigenesis and inappropriate activation of this pathway has been observed in many human cancers. Wnt ligands first bind to Frizzled family of Wnt receptors to form Wnt-Frizzled complexes. Upon binding with the Axin-related protein, Wnt regulates the stability of catenin $\beta$, which is known to play essential roles at cell-cell adherence junctions. Catenin $\beta$ then binds to TCF, a family of transcription factors, inducing the transcription of Wnt target genes, such as c-Myc, c-Jun and Cyclin D1. Both cMyc and c-Jun are oncogenic transcription factors that function in cell cycle progression, apoptosis and cellular transformation. Cyclin D1 is a nuclear protein involved in cell cycle progression in G1/S transition. Activation of these genes contributes to early RCC development. These findings are consistent with the observation that WNT signaling pathway is deregulated during renal carcinoma development. Actin and a variety of actin-binding proteins are also central in the pathway. Remodeling of actins regulates the motility of cells and maintains the cytoskeleton. Cytoskeletal actin disruption is the key factor that triggers apoptosis.

TGF, WNT and cytoskeletal remodeling pathway also contains some objects without previous annotation in ccRCC carcinogenesis, such as ROCK, MEK1, p38, MAPK, axin. These objects could be putative therapeutic targets for novel treatment strategies against ccRCC.

Another pathway preferentially targeted by the DEmiRNAs is Brcal as a transcription regulator, which belongs to the DNA damage category. The pathway map is illustrated in Figure 5 (drawn by MetaCore ${ }^{\mathrm{TM}}$ ). It's evident in Figure 5 that the pathway is enriched with RCC-related objects such as c-Myc, E2F1, Rb protein, IGF1-receptor, VEGF-A and cyclin D1, most of which are predicted targets of miRNAs differentially expressed in ccRCC. The pathway also includes several targets of DE-miRNAs whose association with ccRCC has not been reported before, e.g. Brca1, sp1, sp3, MSH2, p21, GADD45 alpha and stat1. Central in the pathway is the Breast cancer associated gene-1 (Brac1) which plays a central role in DNA repair. BRCA1 is a wellknown tumor suppressor gene in breast cancer and ovarian cancers [36], but its role in other cancer types remains elusive [37]. This is the first study demonstrating the tumor suppressor activity of Brac1 in ccRCC. Brca1 regulates the transcription of proteins at DNA repair pathways via transcription factor $\mathrm{p} 53$, such as mismatch repair protein $\mathrm{MSH} 2$ [38]. Brca1 also participates in cell cycle regulation. In the absence of DNA damage, Brca1 is associated with ZBRK1 in a complex which inhibits transcription of GADD45 alpha. Upon DNA-damage, Brca1 is phosphorylated and dissociated from the Brca1-ZBRK1 repression complex [39]. The released Brca1 stimulates transcription of GADD45 alpha [40]. GADD45 alpha participates in DNA-damage-induced G1/S checkpoint arrest [41] and DNA-damage-induced G2/ $M$ checkpoint arrest [42]. In addition, Brca1 regulates the transcription of some other G1/S checkpoint arrest regulators, e.g. p21 and Cyclin D1. Transcription of p21 [43] may be activated through p53 whereas transcription of Cyclin D1 is regulated via c-Myc [44].

\section{Signatures at pathway level are more consistent}

We performed pair-wise comparison between five datasets at different observation levels, including DEmicroRNA, target gene, GO-MF (Molecular Function), GO-BP (Biological Process), and GeneGo pathway, respectively. For 5 miRNA expression datasets, 10 pairs are available for comparison. P-values by paired t-test are well below 0.05 , indicating that the overlapping percentages at functional level are significantly higher than that at individual DE-microRNA and target gene level.

\section{Discussion}

In this study, we performed systematic and integrative analysis of 5 ccRCC-related microRNA expression datasets, in 


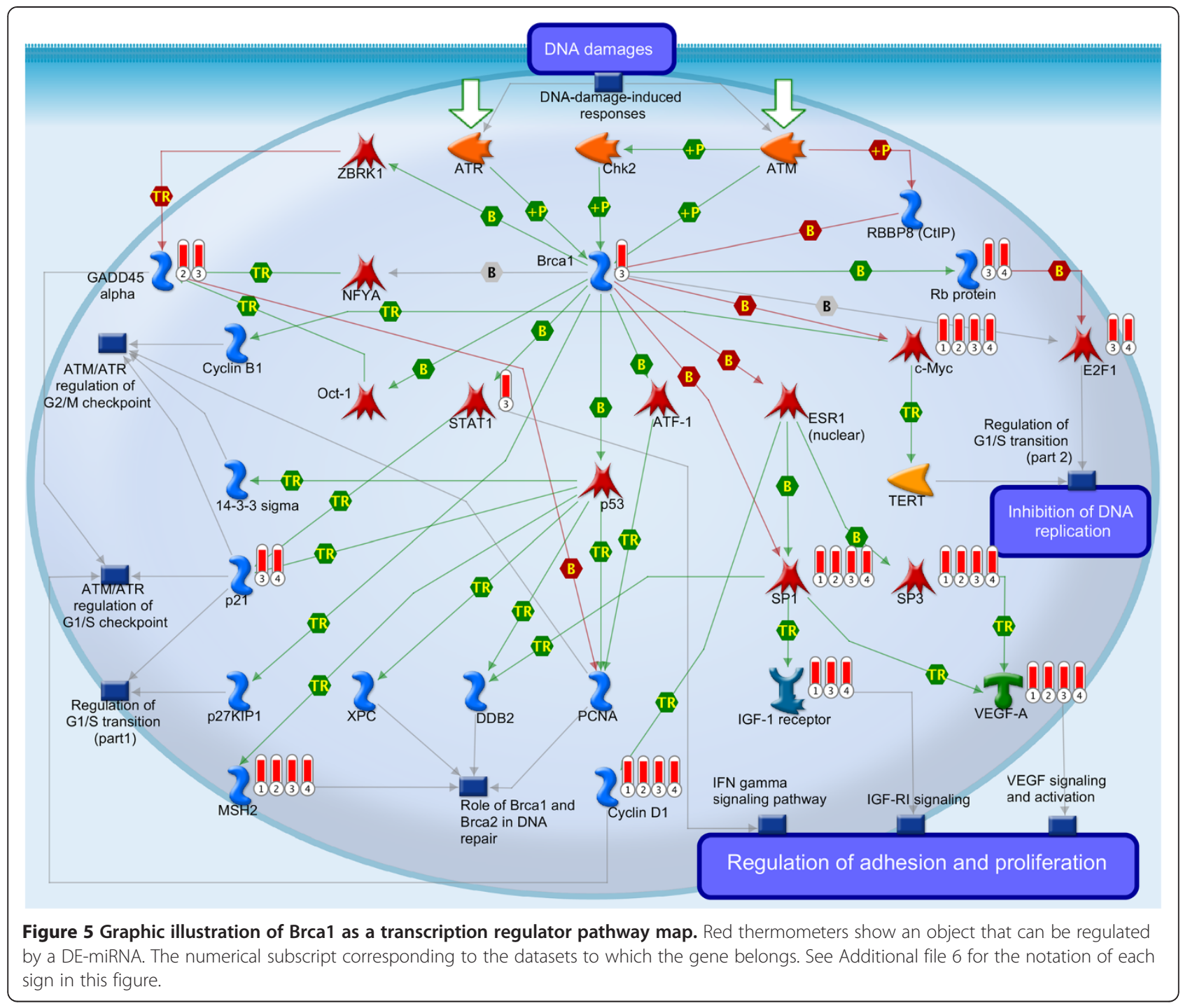

order to find more reliable expression signatures. We incorporated a novel outlier detection algorithm and a functional microRNA prediction model, into an integrative framework which could enhance the reproducibility of results across multiple datasets.

We first applied a new statistics, MOST, to the identification of differentially expressed microRNAs. It was found that some oncogenes have highly heterogeneous activation patterns and are activated in only a small subset of patient samples. This well explains the inter-dataset inconsistency. These subset-specific cancer genes however, cannot be detected with traditional t-tests. As our previous studies $[32,45]$ have indicated, new statistics generally outperform traditional t-statistics and are therefore more competent for cancer data analysis.

We then used POMA to refine the DE-miRNA list by reducing false discoveries. POMA is designed to find the microRNAs with regulatory activity in ccRCC condition.
Those DE-miRNAs without real regulatory activity in the disease were excluded from subsequent analysis. POMA has been employed by our laboratory in the context of prostate cancer (Zhang, unpublished). This model focuses not only on the profile of microRNAs, but also on mRNA transcripts with altered expression in ccRCC. After POMA filtration, final lists of DE-miRNAs are significantly reduced yet improved consistency is observed between the 5 independent datasets.

Finally we obtained a list of $11 \mathrm{DE}$-microRNAs with regulatory roles. Literature mining confirmed that all of these microRNAs have been reported to be deregulated in renal cell carcinoma, which lends credibility to our list.

We further characterized the concordance of our $11 \mathrm{DE}$ miRNA list with the results from NGS (Next-Generation Sequencing) technologies. Next-generation sequencing technology facilitates genome-wide miRNA expression profiling at unprecedented speed and accuracy. It also enables 
discovery of novel miRNAs. After comparison with 3 NGSbased microRNA profiling studies in ccRCC [17-19], we found that our data correlated well with the results of next generation sequencing. For instance, among our 11miRNA panel, up to 9 miRNAs (82\%) were also detected by Osanto et al. [17], except that miR-180 and 660 were not detected. A higher overlap (10 out of 11) was seen comparing with the DE-miRNAs lists by Weng et al. [18], the only mismatch is represented by miR-16-5p. An even better concordance was seen in comparison with the data of Zhou et al. [19]. Here, all of 11 microRNAs were also among the list of deregulated microRNAs.

The comparison with NGS-based data further confirmed that the DE-miRNAs identified by us are authentic cancer related miRNAs in ccRCC and could provide potential biomarkers which await further wet lab validation. Moreover, the general DE-miRNA detection pipeline proposed herein is not limited to ccRCC, but also applicable to a wide range of other diseases.

In order to find novel miRNAs without previous annotation, we also tried to expand the list of POMA filter by lowering the threshold for active miRNA selection. We retrieved active microRNA shared in at least 2 of the 4 mRNA dataset. In this way, a less strict filter with more active miRNA is obtained, which might include some additional novel microRNAs worthy of further investigation. The expanded list of miRNAs is provided as Additional file 7.

The ability of miRNAs to target multiple target genes allows them to induce changes in various pathways and processes, which present a further level of mechanism by which ccRCC may be induced. Overlap analysis at different levels confirms that expression signatures across multiple datasets are more consistent at pathway level than at gene level. It's been recognized cancer is a highly heterogeneous disease.

Single biomarker is unlikely to dictate diagnosis or prognosis success. Consequently, the future of cancer biomarker might rely on coordinated molecular changes instead [46]. As functionally related genes often display a coordinated expression to accomplish their roles in the cell $[47,48]$, one might expect that the inconsistent microRNA lists, when mapped to higher functional levels, could fall within the same functional modules, pathways or networks [49] and become more consistent.

Functional analysis revealed some biological processes which are preferentially targeted by the DE-miRNAs. Interestingly, the top enriched GO terms are mostly involved in cell cycle regulation (e.g., G1/S transition). Aberrant expression of cell cycle regulators could possibly lead to deregulated cell cycle, which is a hallmark of cancer. It's showed that cell cycle checkpoint regulators such as cyclins and cyclin-dependent kinases are coregulated by the DE-miRNAs. For example, miR-16 family is reported to trigger a cell cycle arrest by coordinately suppressing multiple cell cycle regulatory genes [50]. It's worth noting that miR-16 happens to be among the 11 deregulated microRNAs identified in this study. All the evidences above corroborate the validity of the results of the present study.

To evaluate the relevance of the enriched GeneGo pathways in ccRCC, we performed text mining at pathway level as well as object level. Many of the objects that constitute the pathways are known to be critical in the renal carcinogenesis. In addition to the known pathways in RCC tumorigenesis, this study also identified 14 novel ccRCC related pathways. This is the first study demonstrating their relevance in ccRCC. The multiple pathway alterations identified suggest that the miRNAs are potentially regulating many of the necessary steps required by ccRCC development, from changes in the cellular cytoskeleton to regulating cell cycle as well as DNA damage. The cellular functions of these pathways are consistent with the current view on ccRCC pathogenesis. Therefore, these pathways could be a prospective source of novel drug targets and biomarkers. The inhibition of these pathways by synthetic antisense antagomirs provides potential therapeutic interventions in ccRCC.

\section{Conclusions}

In this study we created a bioinformatics framework and applied it to integrative analysis of multiple microRNA expression datasets. The methodology would hopefully improve the reproducibility of miRNAs across independent datasets. The DE-miRNAs and novel pathways identified herein might be candidate biomarkers and drug targets for ccRCC diagnosis and treatment.

\section{Additional files}

Additional file 1: Summary of gene expression datasets used in this study.

Additional file 2: DE-miRNA list after POMA filtration for each dataset.

Additional file 3: Entrez IDs and official symbols of targeting genes for DE-miRNAs of each datasets.

Additional file 4: Enriched GO terms, KEGG pathways and GeneGo pathways shared by 5 datasets.

Additional file 5: Significant GeneGo pathways enriched with both DE-miRNA targets and curated RCC-related genes.

Additional file 6: Legend of the symbols in GeneGo pathway map. This figure provides the notation for each sign in the pathway maps from GeneGo.

Additional file 7: Active microRNA shared in at least 2 of the 4 mRNA dataset.

Competing interests

The authors declare they have no competing interest. 


\section{Authors' contributions}

JC carried out the functional enrichment analysis, performed the statistical analysis and drafted the manuscript. DZ participated in the functional enrichment analysis, and draft the manuscript. WZ designed the POMA algorithm. YT, WY and LG participated in the comparison of outlier detection algorithms. BS conceived of the study, and participated in its design and coordination. All authors read and approved the final manuscript.

\section{Acknowledgements}

We gratefully acknowledge financial support from the National Natural Science Foundation of China grants (91230117, 31170795), the Specialized Research Fund for the Doctoral Program of Higher Education of China (20113201110015), International S\&T Cooperation Program of Suzhou (SH201120), the National High Technology Research and Development Program of China (863 program, Grant No. 2012AA02A601) and Natural Science Foundation for Colleges and Universities in Jiangsu Province (13KJB180021)

\section{Author details}

${ }^{1}$ Center for Systems Biology, Soochow University, Suzhou 215006, China. ${ }^{2}$ School of Chemistry, Biology and Material Engineering, Suzhou University of Science and Technology, Suzhou 215009, China. ${ }^{3}$ Department of Pathology, School of Medicine, Soochow University, Suzhou 215123, China.

Received: 5 March 2013 Accepted: 6 June 2013

Published: 10 July 2013

\section{References}

1. Siegel R, Ward E, Brawley O, Jemal A: Cancer statistics, 2011: the impact of eliminating socioeconomic and racial disparities on premature cancer deaths. CA Cancer J Clin 2011, 61:212-236.

2. Escudier B: Advanced renal cell carcinoma: current and emerging management strategies. Drugs 2007, 67:1257-1264.

3. Singer EA, Gupta GN, Marchalik D, Srinivasan R: Evolving therapeutic targets in renal cell carcinoma. Curr Opin Oncol 2013, 25:273-280.

4. Bartel DP: MicroRNAs: genomics, biogenesis, mechanism, and function. Cell 2004, 116:281-297.

5. Lossos IS, Czerwinski DK, Alizadeh AA, Wechser MA, Tibshirani R, Botstein D, Levy R: Prediction of survival in diffuse large-B-cell lymphoma based on the expression of six genes. N Engl J Med 2004, 350:1828-1837.

6. Allison DB, Cui X, Page GP, Sabripour M: Microarray data analysis: from disarray to consolidation and consensus. Nat Rev Genet 2006, 7:55-65.

7. Kort EJ, Farber L, Tretiakova M, Petillo D, Furge KA, Yang XJ, Cornelius A, Teh BT: The E2F3-Oncomir-1 axis is activated in Wilms' tumor. Cancer Res 2008, 68:4034-4038.

8. Jung M, Mollenkopf HJ, Grimm C, Wagner I, Albrecht M, Waller T, Pilarsky C, Johannsen M, Stephan C, Lehrach H, Nietfeld W, Rudel T, Jung K Kristiansen G: MicroRNA profiling of clear cell renal cell cancer identifies a robust signature to define renal malignancy. J Cell Mol Med 2009, 13:3918-3928.

9. Liu H, Brannon AR, Reddy AR, Alexe G, Seiler MW, Arreola A, Oza JH, Yao M, Juan D, Liou LS, Ganesan S, Levine AJ, Rathmell WK, Bhanot GV: Identifying mRNA targets of microRNA dysregulated in cancer: with application to clear cell Renal Cell Carcinoma. BMC Syst Biol 2009, 4:51.

10. Yi Z, Fu Y, Zhao S, Zhang X, Ma C: Differential expression of miRNA patterns in renal cell carcinoma and nontumorous tissues. $J$ Cancer Res Clin Oncol 2009, 136:855-862

11. White NM, Bao TT, Grigull J, Youssef YM, Girgis A, Diamandis M, Fatoohi E, Metias M, Honey RJ, Stewart R, Pace KT, Bjarnason GA: Yousef GM: miRNA profiling for clear cell renal cell carcinoma: biomarker discovery and identification of potential controls and consequences of miRNA dysregulation. J Urol 2011, 186:1077-1083.

12. Huang $Y$, Dai $Y$, Yang J, Chen T, Yin $Y$, Tang M, Hu C, Zhang L: Microarray analysis of microRNA expression in renal clear cell carcinoma. Eur J Surg Oncol 2009, 35:1119-1123.

13. Chow TF, Youssef YM, Lianidou E, Romaschin AD, Honey RJ, Stewart R, Pace $K T$, Yousef GM: Differential expression profiling of microRNAs and their potential involvement in renal cell carcinoma pathogenesis. Clin Biochem 2010, 43:150-158
14. Juan D, Alexe G, Antes T, Liu H, Madabhushi A, Delisi C, Ganesan S, Bhanot $G$, Liou LS: Identification of a microRNA panel for clear-cell kidney cancer Urology 2009, 75:835-841.

15. Khella HW, White NM, Faragalla H, Gabril M, Boazak M, Dorian D, Khalil B, Antonios H, Bao TT, Pasic MD, Honey RJ, Stewart R, Pace KT, Bjarnason GA, Jewett MA, Yousef GM: Exploring the role of miRNAs in renal cell carcinoma progression and metastasis through bioinformatic and experimental analyses. Tumour Biol 2011, 33:131-140.

16. Berkers J, Govaere O, Wolter P, Beuselinck B, Schoffski P, van Kempen LC, Albersen M, Van den Oord J, Roskams T, Swinnen J, Joniau S, Van Poppel $H$, Lerut $E$ : A possible role for microRNA-141 down-regulation in sunitinib resistant metastatic clear cell renal cell carcinoma through induction of epithelial-to-mesenchymal transition and hypoxia resistance. J Urol 2013, 189:1930-1938.

17. Osanto S, Qin Y, Buermans HP, Berkers J, Lerut E, Goeman JJ, van Poppel H: Genome-wide microRNA expression analysis of clear cell renal cell carcinoma by next generation deep sequencing. PloS One 2012, 7:e38298.

18. Weng L, Wu X, Gao H, Mu B, Li X, Wang JH, Guo C, Jin JM, Chen Z, Covarrubias M, Yuan YC, Weiss LM, Wu H: MicroRNA profiling of clear cell renal cell carcinoma by whole-genome small RNA deep sequencing of paired frozen and formalin-fixed, paraffin-embedded tissue specimens. J Pathol 2010, 222:41-51.

19. Zhou L, Chen J, Li Z, Li X, Hu X, Huang Y, Zhao X, Liang C, Wang Y, Sun L, Shi M, Xu X, Shen F, Chen M, Han Z, Peng Z, Zhai Q, Zhang Z, Yang R, Ye J, Guan Z, Yang H, Gui Y, Wang J, Cai Z, Zhang X: Integrated profiling of microRNAs and mRNAs: microRNAs located on Xq27.3 associate with clear cell renal cell carcinoma. PLoS One 2011, 5:e15224.

20. Kozomara A: Griffiths-Jones S: miRBase: integrating microRNA annotation and deep-sequencing data. Nucleic Acids Res 2011, 39:D152-D157.

21. MacDonald JW, Ghosh D: COPA-cancer outlier profile analysis. Bioinformatics 2006, 22:2950-2951.

22. Lian H: MOST: detecting cancer differential gene expression. Biostatistics 2008, 9:411-418

23. Wu B: Cancer outlier differential gene expression detection. Biostatistics 2007, 8:566-575

24. Tibshirani R, Hastie T: Outlier sums for differential gene expression analysis. Biostatistics 2007, 8:2-8.

25. Wang Y, Rekaya R: LSOSS: Detection of Cancer Outlier Differential Gene Expression. Biomark Insights 2010, 5:69-78.

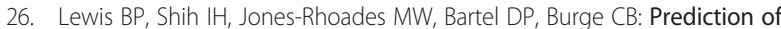
mammalian microRNA targets. Cell 2003, 115:787-798.

27. Krek A, Grun D, Poy MN, Wolf R, Rosenberg L, Epstein EJ, MacMenamin P, da Piedade I, Gunsalus KC, Stoffel M, Rajewsky N: Combinatorial microRNA target predictions. Nat Genet 2005, 37:495-500.

28. John B, Enright AJ, Aravin A, Tuschl T, Sander C, Marks DS: Human MicroRNA targets. PLoS Biol 2004, 2:e363.

29. Xiao F, Zuo Z, Cai G, Kang S, Gao X: Li T: miRecords: an integrated resource for microRNA-target interactions. Nucleic Acids Res 2009 37:D105-D110.

30. da Huang W, Sherman BT, Lempicki RA: Systematic and integrative analysis of large gene lists using DAVID bioinformatics resources. Nat Protoc 2009, 4:44-57

31. Tomlins SA, Rhodes DR, Perner S, Dhanasekaran SM, Mehra R, Sun XW, Varambally S, Cao X, Tchinda J, Kuefer R, Lee C, Montie JE, Shah RB, Pienta KJ, Rubin MA, Chinnaiyan AM: Recurrent fusion of TMPRSS2 and ETS transcription factor genes in prostate cancer. Science 2005, 310:644-648

32. Tang Y, Chen J, Luo C, Kaipia A, Shen B: MicroRNA expression analysis reveals significant biological pathways in human prostate cancer, In 5th IEEE International Conference on Systems Biology, ISB 2011; 2-4 Sept. 2011. Zhuhai, China: EEE Computer Society; 2011:203-210.

33. Zhang $W$, Zhuang $Y$, Jin $X, G$ Go $F$, Shen B: Identification of novel miRNA biomarkers for prostate cancer diagnosis from gene expression data. in press.

34. Ashburner $M$, Ball CA, Blake JA, Botstein D, Butler H, Cherry JM, Davis AP, Dolinski K, Dwight SS, Eppig JT, Harris MA, Hill DP, Issel-Tarver L, Kasarskis A, Lewis S, Matese JC, Richardson JE, Ringwald M, Rubin GM, Sherlock G: Gene ontology: tool for the unification of biology. The gene ontology consortium. Nat Genet 2000, 25:25-29.

35. Kanehisa M, Araki M, Goto S, Hattori M, Hirakawa M, Itoh M, Katayama T, Kawashima S, Okuda S, Tokimatsu T, Yamanishi Y: KEGG for linking genomes to life and the environment. Nucleic Acids Res 2008, 36:D480-484 
36. Miki Y, Swensen J, Shattuck-Eidens D, Futreal PA, Harshman K, Tavtigian S, Liu Q, Cochran C, Bennett LM, Ding W: A strong candidate for the breast and ovarian cancer susceptibility gene BRCA1. Science 1994, 266:66-71.

37. Gao X, Porter AT, Honn KV: Involvement of the multiple tumor suppressor genes and 12-lipoxygenase in human prostate cancer. Therapeutic implications. Adv Exp Med Biol 1997, 407:41-53.

38. Zink D, Mayr C, Janz C, Wiesmuller L: Association of $\mathrm{p} 53$ and MSH2 with recombinative repair complexes during S phase. Oncogene 2002, 21:4788-4800

39. Yun J, Lee WH: Degradation of transcription repressor ZBRK1 through the ubiquitin-proteasome pathway relieves repression of Gadd45a upon DNA damage. Mol Cell Biol 2003, 23:7305-7314.

40. Fan $\mathrm{W}$, Jin $\mathrm{S}$, Tong $\mathrm{T}$, Zhao H, Fan F, Antinore MJ, Rajasekaran B, Wu M, Zhan Q: BRCA1 regulates GADD45 through its interactions with the OCT1 and CAAT motifs. J Biol Chem 2002, 277:8061-8067.

41. Chen IT, Smith ML, O'Connor PM, Fornace AJ Jr: Direct interaction of Gadd45 with PCNA and evidence for competitive interaction of Gadd45 and p21Waf1/Cip1 with PCNA. Oncogene 1995, 11:1931-1937.

42. Jin S, Antinore MJ, Lung FD, Dong X, Zhao H, Fan F, Colchagie AB, Blanck P, Roller PP, Fornace AJ Jr, Zhan Q: The GADD45 inhibition of Cdc2 kinase correlates with GADD45-mediated growth suppression. J Biol Chem 2000, 275:16602-16608.

43. Sancar A, Lindsey-Boltz LA, Unsal-Kacmaz K, Linn S: Molecular mechanisms of mammalian DNA repair and the DNA damage checkpoints. Annu Rev Biochem 2004, 73:39-85.

44. Yin XY, Grove L, Datta NS, Katula K, Long MW, Prochownik EV: Inverse regulation of cyclin B1 by c-Myc and p53 and induction of tetraploidy by cyclin B1 overexpression. Cancer Res 2001, 61:6487-6493.

45. Chen J, Zhang D, Zhang W, Tang Y, Guo L, Shen B: An integrative framework for identifying consistent microRNA expression signatures associated with clear cell renal cell carcinoma, In Systems Biology (ISB), 2012 IEEE 6th International Conference on; 20-22 Aug. 2012. Xian, China: IEEE; 2012:37-42.

46. Chen J, Chen L, Shen B: Identification of Network Biomarkers for Cancer Diagnosis. In Bioinformatics of Human Proteomics. Springer; 2012:257-275.

47. Chen J, Wang Y, Shen B, Zhang D: Molecular signature of cancer at gene level or pathway level? Case studies of colorectal cancer and prostate cancer microarray data. Computational and mathematical methods in medicine 2013, 2013:8.

48. Wang Y, Chen J, Li Q, Wang H, Liu G, Jing Q, Shen B: Identifying novel prostate cancer associated pathways based on integrative microarray data analysis. Comput Biol Chem 2011, 35:151-158.

49. Zhang M, Yao C, Guo Z, Zou J, Zhang L, Xiao H, Wang D, Yang D, Gong $X$, Zhu J, Li Y, Li X: Apparently low reproducibility of true differential expression discoveries in microarray studies. Bioinformatics 2008, 24:2057-2063.

50. Liu Q, Fu H, Sun F, Zhang H, Tie Y, Zhu J, Xing R, Sun Z: Zheng X: miR-16 family induces cell cycle arrest by regulating multiple cell cycle genes. Nucleic Acids Res 2008, 36:5391-5404.

doi:10.1186/1479-5876-11-169

Cite this article as: Chen et al:: Clear cell renal cell carcinoma associated microRNA expression signatures identified by an integrated bioinformatics analysis. Journal of Translational Medicine 2013 11:169.

\section{Submit your next manuscript to BioMed Central and take full advantage of:}

- Convenient online submission

- Thorough peer review

- No space constraints or color figure charges

- Immediate publication on acceptance

- Inclusion in PubMed, CAS, Scopus and Google Scholar

- Research which is freely available for redistribution

Submit your manuscript at www.biomedcentral.com/submit
Biomed Central 